\title{
Residual Generation for Fault Diagnosis of Systems Described by Linear Differential-Algebraic Equations
}

\author{
Erik Frisk and Mattias Nyberg*
}

April 5, 2005

\begin{abstract}
Linear residual generation for DAE systems has been considered. In all results derived, no distinction between input and output signals is done. A complete characterization and parameterization of all residual generators is presented. Further, a condition for fault detectability in DAE systems is given. Based on the characterization of all residual generators, a design strategy for residual generators for DAE systems is presented. Given that a set of faults are detectable, the design strategy will result in a residual generator sensitive to all the detectable faults. Further the residual generator is guaranteed to be of lowest possible order. Special care has been devoted to assure this property also for non-controllable systems.
\end{abstract}

\section{Introduction}

Fault diagnosis consists of detecting and isolating faults acting on a process. In many methods, e.g. structured residuals [6], the concept of residuals play a central role. Commonly, a set of residuals is used where different subsets of residuals are sensitive to different subsets of faults and in this way isolation between faults is possible.

In this paper, residual generation for models described by general linear differential-algebraic equations (DAE) is considered. Previous works on residual generation have all considered more specific classes of models, i.e. transfer functions [6, 22], state-space models [5, 3, 2], or descriptor models e.g. [8, 11]. Since DAE models cover all these classes of models, the methods presented in this paper are applicable to all the three previous cases.

In the context of residual generation, DAE-models are important because they appear in large classes of engineering systems like electrical systems, chemical processes, robotic manipulators, and mechanical systems. For example, in mechanical systems, differential equations arise from equations of motion while algebraic constraints model geometrical constraints. Further, DAE-models are also the result when using a physically based object-oriented modeling approach [14].

\footnotetext{
*Department of Electrical Engineering, Linköping University, SE-581 83 Linköping, Sweden
} \{matny,frisk\}@isy.liu.se 
The approach presented in this paper is an extension of the previous work [5] and one main contribution is a new method for designing residual generators for DAE-models. The method is guaranteed to find residual generators of lowest possible order, and sensitivity to detectable faults is guaranteed. Another main contribution is a criterion for fault detectability in DAE-systems, i.e. a criterion that says if it is at all possible to find any residual generator sensitive to a fault. A help in developing these results, but also a contribution on its own, is a characterization of all possible residual generators has been derived.

Previous works on residual generation for linear DAE systems have all assumed that the model is in descriptor form. As said above, the models considered here (see equation (1)) are more general. However, they can with a straightforward transformation be taken to the descriptor form and therefore, it makes sense to relate the present work to previous works dealing with descriptor models. For descriptor models, two classes of approaches for residual generation can be distinguished: observer-based approaches and parity-spacelike approaches. The approach presented in this paper belongs to the class of parity-space-like approaches.

Observer-based approaches have been studied in $[8,11,12,13,20]$. A limitation of these works compared to the present one is that they all assume observability. Also, the order of the residual generator becomes generally not minimal since the order equals the order of the model. Parity-space-like approaches have been studied in $[21,19,12]$. As in the observer based approaches, also [21] and [19] assume observability. In [12], observability is not assumed but the important problem of decoupling is not considered. The only work discussing minimal order of the residual generator is [21].

\section{Basic Definitions and Problem Formulation}

The aim of this section is to introduce the class of models that is considered, state basic definitions, and precisely state the problem formulation that is explored in the remainder of the paper. The class of models considered is general linear models in the form

$$
H(p) x+L(p) z+F(p) f=0
$$

where $x(t) \in \mathbb{R}^{n_{x}}, z(t) \in \mathbb{R}^{n_{z}}$, and $f(t) \in \mathbb{R}^{n_{f}}$. The matrices $H(p), L(p)$, and $F(p)$ are polynomial matrices in the differentiation operator $p$. The vector $x$ contains all unknown signals, which includes internal system states and unknown inputs such as disturbances. The vector $z$ contains all known signals such as control signals and measured signals and the vector $f$ contains the faultsignals that can affect the system. It is assumed that all signals are piece-wise continuous apart from finitely many impulses and there is no assumption on the initial conditions of any of the signals involved in the model. Also, solutions to (1), and to other differential equations in the presentation as a whole, which are not smooth are considered solutions in the distributional sense unless otherwise stated. Each element of the vector $f$ is associated with one specific fault and when fault $f_{i}$ is not present, its associated element $f_{i}$ in the vector $f$ is zero.

The presentation will be done assuming continuous time, but similar results can be obtained by changing the differentiation operator $p$ to the time-shift operator $q$. For mathematical stringency we will sometimes switch to describe 
matrices in the complex variable $s$ instead of the operator $p$; when describing properties of signals and differential equations we use $p$ but when discussing properties of matrices we use $s$.

The only assumption imposed on the matrices describing the model (1) is that $[H(s) L(s)]$ has full row rank, i.e.

$$
\operatorname{Rank}[H(s) L(s)]=m
$$

where $m$ is the number of equations in the model, i.e. rows in (1). This is a reasonable assumption since it means that there are no linear dependencies in the fault-free model equations.

Now as an example, consider a model given by the following descriptor equations:

$$
\begin{aligned}
E \dot{x} & =A x+B_{u} u+B_{d} d+B_{f} f \\
y & =C x+D_{u} u+D_{d} d+D_{f} f
\end{aligned}
$$

where $y$ is the vector of outputs, $u$ the inputs, $x$ the unknown state-space variable, and $d$ unknown disturbances to be decoupled, and $f$ the faults. Letting $E=I$ in the equations above, an ordinary state-space description is obtained. In general, $E$ can be non-singular and even non-square. Matrices $H(p), L(p)$, and $F(p)$ then becomes

$$
H(p)=\left[\begin{array}{cc}
C & D_{d} \\
-(p E-A) & B_{d}
\end{array}\right], \quad L(p)=\left[\begin{array}{cc}
-I & D_{u} \\
0 & B_{u}
\end{array}\right], \quad F(p)=\left[\begin{array}{l}
D_{f} \\
B_{f}
\end{array}\right]
$$

Thus we have shown how descriptor models fit into the general form (1).

Next we move on to formally define residual generator, what we mean by a detectable fault, and a residual sensitive to a fault. But first, a characterization of all possible measurements from a fault-free model is introduced. This set of measurements is called the fault-free behavior of the system and is formally defined as

$$
\mathcal{M}=\{z \mid \exists x: H(p) x+L(p) z=0\}
$$

which is the same type of models that is used in the behavioral approach to systems theory [18]. Fault detectability as a system property can now be defined using the behavior $\mathcal{M}$.

Definition 1 (Fault Detectability). Fault $i$ is detectable in (1) if there exist signals $f_{i}, x$, and $z$, consistent with (1), where $f_{j}=0$ for $j \neq i$, such that $z \notin \mathcal{M}$.

Earlier, see [16], fault detectability has been defined as the existence of a residual generator such that the transfer function from fault to residual is non zero. The here proposed definition is equivalent to the previous definition, but has the advantage that it is more general in the sense that it covers also the case of a non-linear model description. We are now ready to formally define residual generator.

Definition 2 (Residual Generator). A linear time-invariant filter $r=R(p) z$ with the scalar $r$ as output, is a residual generator for (1) if

$$
z \in \mathcal{M} \Rightarrow \lim _{t \rightarrow \infty} r(t)=0
$$

and its transfer function is proper. 
Note that Definition 2 requires that the limit of $r(t)$ as $t \rightarrow \infty$ is well defined for all $z \in \mathcal{M}$. It might seem that $r$ could contain step functions and impulses, thus making the definition questionable. However, it is shown in Lemma 2 that Definition 2 implies that $z \in \mathcal{M}$ implies that the residual $r$ becomes smooth for all $t$. Since Definition 2 requires $R(s)$ to be proper, a residual generator can always be realized by a state-space description.

The goal is to detect faults included in the vector $f$, while faults that are decoupled, for fault isolation purposes, are included among the unknown signals in $x$. The definition of residual generator above does not require the residual to respond to the faults in the vector $f$. But of course, fault sensitivity is an important property of a residual generator. Fault sensitivity in the residual generator, rather than the system property in Definition 1, is now defined as

Definition 3 (Fault Sensitivity). A residual generator $r=R(p) z$ for $(1)$ is sensitive to fault $i$ if $G_{r f_{i}}(s) \neq 0$.

With these basic definitions we are ready to state the main problem formulation.

Problem formulation: The first objective is to characterize and parameterize all residual generators for a model (1), using minimal number of parameters. Since the goal of residual generation is to detect faults, there is a need to determine if this is at all possible based on the model (1). Therefore, a second objective is to find detectability conditions for models in the form (1). The final main objective is to, given a set of detectable faults, derive a design method that finds residual generators sensitive to these faults. In the design method we are especially interested in finding residual generators of minimal order. The reason for using minimal order residual generators is to save valuable computer memory in embedded systems, and also that these should give the best numerical on-line performance.

\section{A Polynomial Characterization of All Resid- ual Generators}

In this section we will find a general expression that characterizes and parameterizes all residual generators for a given model (1). The parameterization should be minimal in the sense that it uses a minimal number of parameters. To do this we first introduce the basic idea of how residual generators can be constructed and then the characterization is proven.

First, let the rows of $N_{H}(s)$ form an irreducible polynomial basis for the left null-space of the matrix $H(s)$. If we let $f=0$ and multiply (1) from the left with $N_{H}(p)$, we obtain the expression $N_{H}(p) L(p) z=0$. In this equation the influence of the unknown signals $x$, such as disturbances, has been decoupled. However, this equation defines the same set of trajectories of $z$ as the original model (1) with $f=0$, see [18]. Thus the set $\mathcal{M}$ in (5) can alternatively be written as

$$
\mathcal{M}=\left\{z \mid N_{H}(p) L(p) z=0\right\}
$$

see Lemma 1. By picking one row in $N_{H}(p) L(p)$, or a linear combination of rows specified by a row vector $\gamma(p)$, we obtain a so called parity relation 
$\gamma(p) N_{H}(p) L(p) z=0$. By adding stable dynamics $d(p)$ of sufficiently high order we obtain a residual generator with transfer operator

$$
R(p)=d^{-1}(p) \gamma(p) N_{H}(p) L(p)
$$

and that can be realized by an explicit state-space description. This is our basic idea of how residual generators can be constructed for models in the form (1). Later in Section 5, this design method is developed further and special care is devoted to fault sensitivity and the order of the obtained residual generator.

With these principles in mind, we will now investigate if the expression (6) is a general expression characterizing all residual generators where the scalar polynomial $d(s)$ and the row-vector $\gamma(s)$ are the parameters. For this consider the model equation

$$
(p+1) z_{1}-(p+1) z_{2}=0
$$

where $H(s)=0$ and $L(s)=[s+1 s+1]$. Then $R(p)=[1-1]$ is a residual generator since solving the differential equation (7) results in

$$
r=R(p) z=z_{1}-z_{2}=e^{-t}\left(z_{1}(0)-z_{2}(0)\right)
$$

which clearly satisfies Definition 2 . However, since in this example $N_{H}(p) L(p)=$ $[p+1 p+1]$, there are no choices of $d(s)$ and $\gamma(s)$ such that $(6)$ holds. Note that the choice $d(s)=(s+1)$ and $\gamma(s)=1$ will not fulfill $(6)$ since $(p+1)^{-1}[p+$ $1 p+1] \neq\left[\begin{array}{ll}1 & 1\end{array}\right]$.

Thus, we have shown that the expression (6) is not a general characterization of all residual generators. The reason why (6) can not characterize the residual generator (8) originates from the fact that the system (7) is not controllable. Controllable here refers to a generalized controllability definition valid also for non-causal DAE-systems [18]. In the context of this paper, a system is controllable if the matrix $[H(s) L(s)]$ has full rank for all $s$.

In general it holds that for systems with non-controllable stable modes, all residual generators can not be characterized by (6). We will now give an explanation to this. If $[H(s) L(s)]$ has a stable zero for some $s=s_{0}$, also $Q(s) \triangleq N_{H}(s) L(s)$ will have a zero for $s=s_{0}$. The matrix $Q(s)$ can therefore be factorized according to $Q(s)=Q_{s t a b}(s) Q_{r}(s)$ where $Q_{s t a b}(s)$ is a square and full-rank matrix with only strictly stable zeros, and $Q_{r}(s)$ has no strictly stable zeros. Since $Q(p) z=Q_{s t a b}(p) Q_{r}(p) z=0$ and $Q_{s t a b}(s)$ is full-rank and Hurwitz, the signal $Q_{r}(p) z$ must be smooth and go asymptotically to zero [18]. All rows in $Q_{r}(s)$ can therefore be used to form residual generators. However, not all rows of $Q_{r}(s)$ can be written as $\gamma(s) N_{H}(s) L(s)$ and this is the answer to why (6) does not work as a general characterization of all residual generators. Based on the matrix $Q_{r}(s)$, we give in the following main theorem a correct alternative to $(6)$.

Theorem 1 (Characterization). Let $Q_{r}(s)$ be a matrix such that $Q_{s t a b}(s) Q_{r}(s)=$ $N_{H}(s) L(s)$ where $Q_{s t a b}(s)$ is a square and full-rank matrix with only strictly stable zeros and $Q_{r}(s)$ has no strictly stable zeros. A proper filter with transfer operator $R(p)$ is a residual generator for (1) if and only if there exists a polynomial row-vector $\gamma(s)$ and a stable polynomial d(s) such that

$$
R(p)=d^{-1}(p) \gamma(p) Q_{r}(p)
$$


Proof. The if-part is proven by the discussion above the theorem and for the only-if part, Lemma 3 gives that $z \in \mathcal{M} \Rightarrow \lim _{t \rightarrow \infty} K(p) z(t)=0$. This gives that the scalar denominator $d(s)$ must be stable since if $v=K(p) z$ it holds that $d(p) r=v$ and when $z \in \mathcal{M}$ both $r$ and $v$ goes to zero. Also, using Lemma 4 gives that there exists a $\gamma(s)$ such that $K(s)=\gamma(s) Q_{r}(s)$ which implies that $R(p)=d^{-1}(p) \gamma(p) Q_{r}(p)$ which ends the proof.

Since the matrix $[H(s) L(s)]$ has full row rank, also the matrix $Q_{r}(s)$ also has full row rank, which in turn implies that $\gamma(s)$ will contain a minimal number of elements. Thus, the parameterization (9) will be minimal.

It can be verified that in the example $(7), Q_{r}(s)=[1-1]$ and therefore (8) can be written in the form (9). Before giving the proof to Theorem 1, a few connections of the result above to the well known notion of parity functions [6] is outlined. First, if no factorization of $Q(s)$ is done, any expression in the form $\gamma(p) Q(p) z$ is a standard parity function, i.e. $z \in \mathcal{M}$ implies that $\gamma(p) Q(p) z=0$ for all $t$. However, when performing the factorization $Q(s)=Q_{s t a b}(s) Q_{r}(s)$, an expression $\gamma(p) Q_{r}(p) z$ may not equal 0 for all $t$, it will however go to 0 as $t \rightarrow \infty$ with a rate of decay according to the dynamics of $Q_{s t a b}(s)$. Thus, for a system with non-controllable stable modes and a residual generator formed according to (9), the residual will not be 0 for all $t$ in a fault free case; it will go to 0 . However, this is typically the case for a proper residual generator constructed with any other approach, e.g. see observer-based residual generators [2]. The difference is that when $Q_{s t a b}(s)$ is not factored out, the decay rate is completely determined by the design choice of $d(s)$, and when $Q_{s t a b}(s)$ is factored out, the decay rate is determined by the dynamics of both $d(s)$ and $Q_{s t a b}(s)$.

\section{Fault Detectability}

A main objective of the paper is design of residual generators that are sensitive to a specified set of faults. Sensitivity to a fault as defined in Definition 3 is a property of the residual generator but is in fact closely related to the system property fault detectability as defined in Definition 1. The exact relationship is revealed by the theorem below.

Theorem 2. There exists a residual generator for (1) sensitive to all faults in $f$ if and only if all faults in $f$ are detectable in (1).

Proof. If part: The fact that a fault $f_{i}$ is detectable means that

$$
\exists z, f_{i}: Q(p) z=-N_{H}(p) F_{i}(p) f_{i} \neq 0
$$

Let $t_{0}$ be a time such that $N_{H}(p) F_{i}(p) f_{i}(t) \neq 0$ for some $t>t_{0}$. Let $\bar{f}(t)=0$ for $t<t_{0}$ and $\bar{f}(t)=f_{i}(t)$ for $t \geq t_{0}$. From the fact that $[H(s) L(s)]$ has full row rank, it follows that for any signal $f$ there exist $x$ and $z$ such that the model (1) is fulfilled. Thus, for $\bar{f}$ there exists $z$ such that $Q(p) z=-N_{H}(p) F_{i}(p) \bar{f}$. Since $\bar{f}(t)=0$ for all $t<t_{0}$, it holds that $N_{H}(p) F_{i}(p) \bar{f}(t)=0$ for $t<t_{0}$. From above we also know that $N_{H}(p) F_{i}(p) \bar{f}(t) \neq 0$ for some $t>t_{0}$. Therefore it holds that $Q(p) z=0$ for all $t<t_{0}$ and $N_{H}(p) F_{i}(p) \bar{f}(t) \neq 0$ for some $t>t_{0}$. With a constant row vector $\gamma_{i}$, we can now obtain a $\gamma_{i} Q(p) z$ such that this property is preserved. By having done this for all faults in $f$, we choose a $\gamma=\sum_{i} \gamma_{i}$. 
When considering one fault at a time, all other fault signals $f_{i}$ are considered to be zero. Therefore, for each fault in $f$ it holds that with a $\bar{f}$, as derived above, it holds that $\gamma Q(p) z=0$ for all $t<t_{0}$ and $\gamma Q(p) z=\gamma N_{H}(p) F_{i}(p) \bar{f}(t) \neq 0$ for some $t>t_{0}$. For each such $\bar{f}$ the residual equation $d(p) r=\gamma Q(p) z$ has a solution $r$ where $r(t)=0$ for all $t<t_{0}$ and $r(t) \neq 0$ for some $t>t_{0}$. Thus, we have shown that there is a residual generator $R(p)$ that is sensitive to each fault in $f$.

Only-if part: From Theorem 1 we know that the residual generator $R(p)$ can be written as $d(p) r=\gamma(p) Q_{r}(p) z$. The fact that the residual generator is sensitive to a fault $f_{i}$ means that $(10)$ and that there is a time $t_{1}$ such that $\gamma(p) Q_{r}(p) z(t)=0$ for $t<t_{1}$ and $\gamma(p) Q_{r}(p) z(t) \neq 0$ for some time $t>t_{1}$.

Assume now that for all $t$, the homogeneous equation $Q_{\text {stab }}(p) Q_{r}(p) z(t)=0$ holds. The fact that $Q_{s t a b}(s)$ has full rank limits the type of solutions $v=$ $Q_{r}(p) z(t)$ to sums of terms of the type $q_{j}(t) \exp \left(\tau_{j} t\right)$ where $q_{j}(t)$ is a polynomial in $t$. This together with $\gamma(p) Q_{r}(p) z(t)=0$ for $t<t_{1}$ implies that $\gamma(p) Q_{r}(p) z(t)=0$ also for $t \geq t_{1}$. This contradiction means that $v=Q_{r}(p) z(t)$ can not be a solution to the differential equation $Q_{s t a b}(p) v=0$. Thus we have shown that $Q_{s t a b}(p) Q_{r}(p) z \neq 0$ which says that $f_{i}$ is detectable.

Thus, before starting the design of a residual generator, it is natural to investigate if the faults are detectable.

Since Definition 1 deals with detectability of one single fault, testing detectability of a set of faults will be done by considering one fault at a time. The intuitive main result on fault detectability can now be stated.

Theorem 3. Fault $i$ is detectable in (1) if and only if

$$
\operatorname{Rank}\left[H(s) F_{i}(s)\right]>\operatorname{Rank} H(s)
$$

Proof. If fault $i$ is detectable, there exists $f_{i}, x$, and $z$, consistent with (1) and $z \notin \mathcal{M}$. Then multiplying (1) from the left with $N_{H}(p)$ results in $Q(p) z=$ $-N_{H}(p) F_{i}(p) f_{i}$. Since $z \notin \mathcal{M}$, it holds according to Lemma 1 , that $Q(p) z \neq 0$. This implies that $N_{H}(p) F_{i}(p) f_{i} \neq 0$ and it must therefore hold that $N_{H}(s) F_{i}(s) \neq$ 0 which is equivalent to $(11)$.

If $N_{H}(s) F_{i}(s) \neq 0$, there exists a signal $f_{i}$ such that $N_{H}(p) F_{i}(p) f_{i} \neq 0$. For this signal $f_{i}$, because of (2) there exist also signals $x$ and $z$ such that (1) is fulfilled. Multiplying (1) from the left with $N_{H}(p)$ results in $Q(p) z=$ $-N_{H}(p) F_{i}(p) f_{i} \neq 0$. Lemma 1 then gives that $z \notin \mathcal{M}$ which means that the fault is detectable.

In principle, this condition assures that when decoupling the unknown signals $x$, the fault $f_{i}$ should not also be decoupled. Detectability conditions have been presented in previous literature. e.g. see $[4,15]$. For a survey and comparison of detectability conditions for systems in state-space form and transfer function form, see [16]. The above criterion is different from previous criterions in the way that it is valid for any kind of linear DAE system. However, if for example state-space systems are considered, the criterion (11) becomes equivalent to previous published criterions, see [16]. 


\section{Design of Residual Generators}

When it has been concluded that the faults considered are detectable it is time to start the design of a residual generator to be used in the diagnosis system. The basic principle for residual generation has already been given in Theorem 1 . However, the parameter vector $\gamma(s)$ is in Theorem 1 a free parameter, and it will here be shown how this parameter should be chosen to obtain a residual generator that is sensitive to all detectable faults. Further, the completeness property in Theorem 1 will here make it possible to also guarantee that the obtained residual generator is of lowest possible order.

First a brief comment on decoupling and fault isolation. For isolation purposes, a subset of the faults may need to be decoupled in each residual. To decouple a fault $f_{i}$ in the residual, temporarily consider $f_{i}$ to be an unknown signal in $x$, i.e. modify $H(s)$ to be

$$
\left[H(s) \quad F_{i}(s)\right]
$$

and remove the corresponding column $F_{i}(s)$ from $F(s)$. Decoupling of more than one fault is a straightforward generalization of this procedure. Now, assume that the model is in the form (1), any fault to be decoupled is considered an element in vector $x$ and that each fault in $f$ is detectable. The proposed design method is then as follows:

1. Compute an irreducible polynomial basis $N_{H}(s)$ for the left null-space of $H(s)$ and form $Q(s)=N_{H}(s) L(s)$. If the model has no $H(s)$, let instead $Q(s)=L(s)$.

2. Factorize $Q(s)$ as $Q(s)=Q_{s t a b}(s) Q_{r}(s)$, where $Q_{s t a b}(s)$ is square and full rank, and $Q_{r}(s)$ has no strictly stable zeros and $Q_{\text {stab }}(s)$ has no unstable zeros.

3. If $Q_{r}(s)$ is not row-reduced, find a unimodular matrix $U(s)$ such that $U(s) Q_{r}(s)$ is row-reduced and rows ordered, from top to bottom, with increasing row degrees.

4. Choose a constant row vector $\gamma^{\prime}$, of minimal length, such that

$$
\left[\begin{array}{llll}
\gamma^{\prime} & 0 & \ldots & 0
\end{array}\right] U(s) \operatorname{adj} Q_{s t a b}(s) N_{H}(s) F_{i}(s) \neq 0
$$

Let $\gamma(s)=\left[\begin{array}{llll}\gamma^{\prime} & 0 & \ldots & 0\end{array}\right] U(s)$.

5. Choose a stable and scalar polynomial $d(s)$ such that $\operatorname{deg} d(s)=\operatorname{deg} \gamma(s) Q_{r}(s)$.

6. Find a minimal realization of the filter $R(p)=d^{-1}(p) \gamma(p) Q_{r}(p)$.

The existence of a $\gamma^{\prime}$ in step 4 and other important properties of the method are proven in the following theorem.

Theorem 4. If each fault in the vector $f$ is detectable in (1), the design method above will find a residual generator $R(p)$ for $(1)$. Further, the residual generated by $R(p)$ will be sensitive to all the faults in $f$, and $R(p)$ will be of lowest order. $\diamond$ 
Proof. Since $R(p)=d^{-1}(p) \gamma(p) Q_{r}(p), d(s)$ is stable, $R(p)$ is according to Theorem 1 a residual generator.

Since all faults in $f$ are detectable, we know from Theorem 3 that for all $i$ it holds that $N_{H}(s) F_{i}(s) \neq 0$. Since the square matrix $U(s) \operatorname{adj} Q_{s t a b}(s)$ has full rank it holds that for all $i, U(s) \operatorname{adj} Q_{s t a b}(s) N_{H}(s) F_{i}(s) \neq 0$. The existence of a $\gamma^{\prime}$ in step 4 is therefore guaranteed which implies that the residual will be sensitive to all faults in $f$.

We will now show that $R(p)$ is of lowest possible order. From Theorem 1 it follows that any other residual generator sensitive to the same set of faults can be written as $\bar{d}^{-1}(p) \alpha(p) Q_{r}(p)$. Let $m$ be the number of elements in the vector $\gamma^{\prime}$. Let $\alpha^{\prime}$ be defined by $\left[\alpha^{\prime}(s) 0 \ldots 0\right]=\alpha(s) U^{-1}(s)$ where $\alpha^{\prime}(s)$ has $n$ number of elements and the last element of $\alpha^{\prime}(s)$, denoted $\alpha_{n}^{\prime}(s)$, is non-zero. Note first that $n \geq m$. Otherwise $\gamma^{\prime}$ can not have minimal number of elements. Next note that $\operatorname{deg}\left[\alpha^{\prime}(s) 0 \ldots 0\right] U(s) Q_{r}(s)$ is, according to the predictable degree property [10], at least as large as $\operatorname{deg} \alpha_{n}^{\prime}(s)$ plus the degree of the $n$ :th row of $U(s) Q_{r}(s)$. The $n$ :th row of $U(s) Q_{r}(s)$ has a degree higher or equal to the $m$ :th row of $U(s) Q_{r}(s)$. Further, since $\gamma^{\prime}$ is constant, and the rows of $U(s) Q_{r}(s)$ are ordered in increasing row order, the predictable degree property gives that $\operatorname{deg}\left[\gamma^{\prime} 0 \ldots 0\right] U(s) Q_{r}(s)$ is always equal to the degree of the $m$ :th row. All this means that $\operatorname{deg} \alpha(s) Q_{r}(s) \geq \operatorname{deg} \gamma(s) Q_{r}(s)$. This implies that any other residual generator has an order higher than $\operatorname{deg} \gamma(s) Q_{r}(s)$ which is the order of $R(p)$.

In some cases it is desirable to form the frequency response of a fault. Discussion on this is not included in the design method but the freedom available in the parameters $\gamma^{\prime}$ and $d(s)$ can be used for this. In addition, the residual can of course also be post-filtered by a low-pass filter.

Step 1, 2, and 3 contain operations that involve non-trivial manipulations of polynomial matrices. These operations can for example be carried out using Polynomial Toolbox for Matlab [1], which contains functions for each of these steps. A detailed discussion about underlying algorithms can be found in [7]. Using these algorithms, the first step of deriving the basis for the null-space is numerically sound. Also the second and third step are numerically sound as long as the zeros of $Q(s)$ have low multiplicities and if $Q(s)$ is of reasonable size [7]. This can normally be expected in this case since the number of rows in $Q(s)$ equals the number of redundant equations in the model and the zeros of $Q(s)$ corresponds to uncontrollable modes of the model.

Some of the steps in the design method, in particular steps 2 and 3, may in many cases be omitted depending on properties of the particular model considered rendering a simplified design algorithm. For example, if the system is controllable the matrix $[H(s) L(s)]$ will be irreducible which implies that $Q(s)$ has no zeros. Thus step 2 becomes unnecessary, and $Q_{r}(s)$ and $Q_{s t a b}(s)$ in the remaining steps can be chosen as $Q_{r}(s)=Q(s)$ and $Q_{s t a b}(s)=I$. Also, for state-space models (and many other models) controllability is sufficient for $Q(s)$ to be both irreducible and row-reduced [5], i.e. also step 3 can be omitted.

\section{Example}

Consider the cart on rail illustrated in Fig. 1. The back wheel-pair is powered and both wheel pairs have suspension. The force applied at the back wheel- 


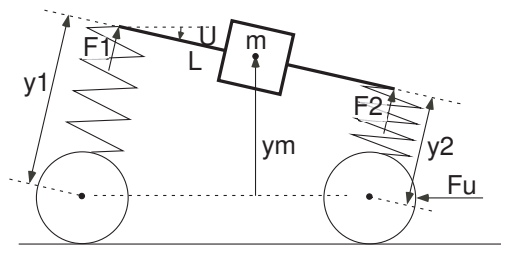

Figure 1: Cart on rail.

pair is $F_{u}$, the vertical position of the cart is $y_{m}$, the other variables $\theta, y_{1}$, $y_{2}, F_{1}$, and $F_{2}$ are indicated in the figure. It is assumed that the force $F_{u}$ is controlled by a control signal $u$, and that the distances $y_{1}$ and $y_{2}$ are measured by sensors $y_{1 s}$ and $y_{2 s}$ respectively. The task in this example is to diagnose faults in the actuator and also in the sensors. These faults are modeled by additive fault-signals $f_{1 s}, f_{2 s}$, and $f_{u}$. A linearized model for this cart is given by the equations

$$
\begin{aligned}
& y_{m}=y_{1}-l \theta, \quad y_{1 s}=y_{1}+f_{1 s}, \quad F_{2}=-k y_{2}-\mu \dot{y}_{2} \\
& y_{m}=y_{2}+l \theta, \quad F_{u}=u+f_{u}, \quad J \ddot{\theta}=l F_{1}-l F_{2}+(C-m g /(2 k)) F_{u} \\
& m \ddot{y}_{m}=F_{1}+F_{2}, \quad F_{1}=-k y_{1}-\mu \dot{y}_{1}, \quad y_{2 s}=y_{2}+f_{2 s}
\end{aligned}
$$

The constants are assumed to have values $\mu=0.1, k=0.5, l=1, J=0.02$, and $m=5, C=0.3$.

The design goal is now to find a residual generator that is sensitive to $f_{2 s}$ and $f_{u}$. Further, the fault $f_{1 s}$ should be decoupled for fault isolation purposes. Then the vector of known signals is $z=\left[\begin{array}{lll}y_{1 s} & y_{2 s} & u\end{array}\right]^{T}$ and the vector of unknown signals $x=\left[\begin{array}{llllllll}\theta & y_{m} & y_{1} & y_{2} & F_{1} & F_{2} & F_{u} & f_{1 s}\end{array}\right]^{T}$. The vector of faults to be detected is $f=\left[f_{2 s} f_{u}\right]^{T}$. Using all equations (13), it is now straightforward to set up the matrices $H(s), L(s)$, and $F(s)$. All matrices have 9 rows and the number of columns will match the dimensions of the vectors $x, z$, and $f$ respectively.

The design will now be described and the calculations have been performed using Matlab and Polynomial Toolbox [1]. Before we start the design, we check that the design goal is at all achievable. This means that faults $f_{2 s}$ and $f_{u}$ should be detectable. By using the simple rank condition (11) for each fault respectively, it can be checked that they are detectable. Theorem 4 now tells us that the design will result in a residual generator, of minimal order, sensitive to both faults $f_{2 s}$ and $f_{u}$.

Following the design method, we start in Step 1 by calculating the basis $N_{H}(s)$. The matrix $Q(s)$ is in Step 2 then formed as $Q(s)=N_{H}(s) L(s)$. The matrix $Q(s)$ has zeros in $s=-0.02 \pm i 0.4468$ and the factorization $Q(s)=$ $Q_{s t a b}(s) Q_{r}(s)$ then consists of

$Q_{s t a b}(s)=\left[s^{2}+0.04 s+0.2\right], Q_{r}(s)=\left[\begin{array}{lll}0 & 0.00041 s^{2}+0.0041 s+0.0205 & -1\end{array}\right]$

Since $Q_{r}(s)$ has only one row it is trivially row-reduced which implies that Step 3 becomes unnecessary. In Step 4 we set up the matrix

$U(s) \operatorname{adj} Q_{s t a b}(s) N_{H}(s) F(s)=10^{-2}\left[0.04 s^{4}+0.4 s^{3}+2 s^{2}+0.16 s+0.4 \quad-98 s^{2}-3.9 s-20\right]$

Since $Q_{r}(s)$ has only one row, selection of $\gamma$ is trivial and is set to $\gamma=1$. In Step 4 we see that $\operatorname{deg} \gamma Q_{r}(s)=2$ and $d(s)$ is then selected as, for example, 
$d(s)=(s+3)^{2}$. This means finally that $R(p)$ becomes

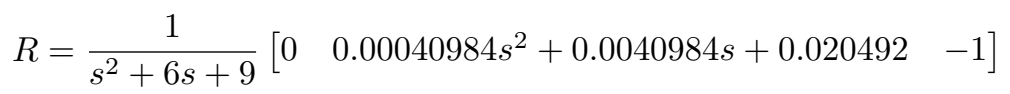

According to Theorem 4 this residual generator has now the lowest possible order among residual generators sensitive to the faults. It can be seen that without the factorization (14), lowest order would not have been achieved.

This design example has shown the case where one residual is designed where one fault is decoupled. If instead full isolation is of interest, it would be desirable to have three residuals where in each, one of the faults is decoupled. The only principle difference compared to the design shown above is that vectors $x$, and $f$ need to be redefined for each design and the matrices $H(s)$ and $F(s)$ must also be changed accordingly.

\section{Illustrative Example}

Here is a second example used to illustrating the design procedure. The example is based on, but not identical to, a descriptor model described in [9] and used for diagnosis in [8]. The model is an idealized description of a three-link planar manipulator/robot seen in Figure 2. The process works by moving the end

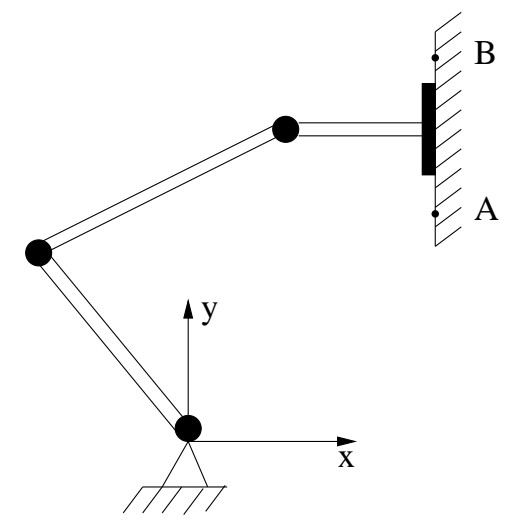

Figure 2: An idealized three-link manipulator

effector repeatedly, e.g. cleaning a facade. The manipulator is equipped with three actuators that can apply torques at all three joints. Three sensors is used measuring the height of the end effector, the contact force in the x-direction, and a tracking signal. The fault-free model is stated on descriptor form in [9] where also numerical values for model parameters can be found. The model has 8 states: Cartesian coordinates of the end effector (3 states), derivatives of the Cartesian coordinates (3 states), and two Lagrangian multipliers.

In Section 1, the benefits and consequences of component and object-oriented based modeling is discussed. To illustrate these matters, an additional sensor and two fault models are added. The first state $x_{1}$ is the height of the end effector. Now, assume that an accelerometer is also attached to the end-effector, thus $\ddot{x}_{1}(t)$ is measured. Also, two faults are modeled, one fault acting on the first actuator and a sensor fault on the first sensor. Collecting the original 
model, the additional sensor, and the fault models results in the following model description:

$$
\begin{aligned}
p E x(t) & =A x(t)+B_{u} u(t)+B_{1} f_{1}(t) \\
y_{1}(t) & =x_{2}(t)+f_{2}(t) \\
y_{2}(t) & =x_{7}(t) \\
y_{3}(t) & =x_{8}(t) \\
y_{4}(t) & =p^{2} x_{1}(t)
\end{aligned}
$$

where $B_{1}$ is equal to the first column in $B_{u}$. Note that the new sensor-equation (15e) was straightforward to introduce and no parts of the model equations had to be modified. In a state-space/descriptor setting, additional states would have been necessary all depending on the original system. This illustrates the modeling principles discussed in Section 1 regarding modularity, object-oriented, and component based modeling. If the model was to be used for control, perhaps a state-space/descriptor formulation would have been preferable, but for diagnosis applications this general class of models is equally useful.

Now, the model (15) can easily be stated on form (1) with unknown variables $x(t)$, and observables $z(t)=\left[y^{T}(t) u^{T}(t)\right]^{T}$. It is obvious that the model above is not on state-space or descriptor form. Although it is possible to transfer the model to a descriptor form, it is not a trivial operation and with the proposed design algorithm, model equations (15) can be used directly without any additional transformations.

\subsection{Designing Residual Generators}

In this example, two residual generators $R_{1}(p)$ and $R_{2}(p)$ are to be designed, used for detection and isolation of the two faults $f_{1}$ and $f_{2}$. In the residual generators fault $f_{1}$ and $f_{2}$ should be decoupled in $R_{1}(p)$ and $R_{2}(p)$ respectively, i.e. residual $r_{1}$ should only react to fault $f_{2}$ and vice versa. Before constructing the residual generators $R_{1}(p)$ and $R_{2}(p)$, the detectability condition from Section 4 can now be applied. It is seen that both $f_{1}$ and $f_{2}$ are detectable when the other fault is decoupled.

Then the design of $R_{1}(p)$ and $R_{2}(p)$ starts by computing rank and greatest left divisor of the matrix $[H(s) L(s)]$. It is seen to have full row-rank and no zeros, which together with the discussion at the end of Section 5 gives that some of the steps from the design algorithm can be omitted. Full Matlab code for design of a residual generator is given below.

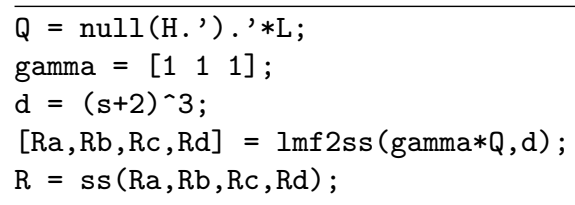

The code uses two toolboxes, [1] and control toolbox. Note that all operations are standard operations in these toolboxes and no diagnosis-specific algorithms need to be developed and/or written. The model matrices $H(s)$ and $L(s)$ are assumed to be defined and the resulting residual generator $R(s)$ is in state-space form (an LTI object in control toolbox). 
All steps are self explanatory except maybe line 4 which transforms a left MFD to a state-space description. For both designs, $Q(s)$ is a polynomial matrix of degree 2 with 3 rows, i.e. there exists exactly 3 linearly independent consistency relations where $f_{1}$ respectively $f_{2}$ are decoupled. The two design choices that exists in the design are the choice of $\gamma(s)$ and the choice of denominator $c(s)$ such that the residual generator is stable and realizable on state-space form. Here these choices are done ad-hoc since no additional design specifications are given. The row vector $\gamma(s)$ is selected to use all consistency relations with $\gamma(s)=\left[\begin{array}{lll}1 & 1 & 1\end{array}\right]$ and all poles of the residual generator, i.e. zeros of $c(s)$, are placed in $s=-2$. For the residual generator to be proper, it turns out that the denominator polynomial need to be of at least degree two, here a third order denominator polynomial is used.

A first step when evaluating the designs is to validate that the desired decoupling properties are satisfied. Computing the sizes of the transfer functions from the decoupled faults to residuals in Matlab we get $\left\|G_{r_{1} f_{1}}(s)\right\|_{\infty}=0$ and $\left\|G_{r_{2} f_{2}}(s)\right\|_{\infty}=0$. This verifies that faults $f_{1}$ and $f_{2}$ are decoupled in $r_{1}$ and $r_{2}$ respectively according to design specifications. Figure 3 shows transfer functions from faults to the residuals, and it is clear that each residual will react according to design specifications. Also, the cut-off frequency is, as specified by the denominator polynomial, approximately $2 \mathrm{rad} / \mathrm{s}$. One important thing that

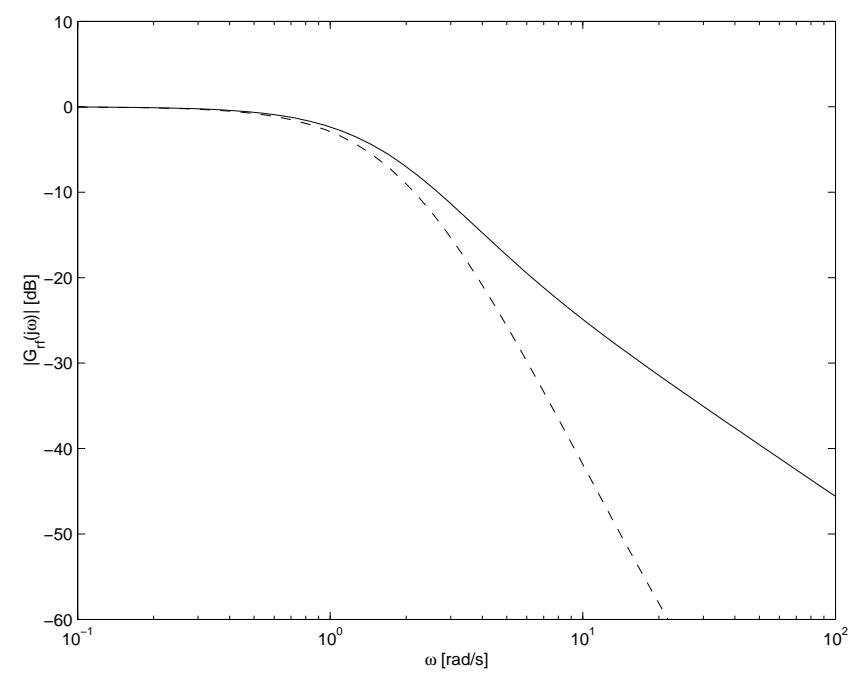

Figure 3: Transfer function from fault $f_{2}$ to residual $r_{1}$ (solid line), and from fault $f_{1}$ to residual $r_{2}$ (dashed line).

has not been mentioned is numerical performance of the design procedure. This is not pursued further here, interested readers is referred to previous works on both state-space and descriptor models that shows good numerical performance (compared to other approaches) on example models [5]. 


\section{Conclusions}

Linear residual generation for DAE systems in the form (1) has been considered. This is an important model class since physically based models are often described by DAE systems. The more common model classes state-space form, descriptor systems, and transfer functions can all be considered as special cases of (1) and all results presented are valid also for these cases.

Throughout the presentation, no distinction between input and output signals have been done. In this way we have shown that what is important for diagnosis, and in particular residual generation, is to distinguish between known and unknown signals.

The notions of residual generator, fault detectability, and fault sensitivity have been formally defined in the framework of DAE models. Then a complete characterization and parameterization of all residual generators has been presented. A condition for fault detectability in DAE systems has been derived and the notions of fault detectability and fault sensitivity has been linked together.

Based on the characterization of all residual generators, a design method for residual generators for DAE systems was presented. Given a set of detectable faults, the design method results in a residual generator sensitive to all detectable faults. Further, the residual generator is guaranteed to be of lowest possible order.

With the aim to make all the results as general as possible, we have devoted special care to non-controllable systems. Without this attention, the characterization derived would not have been complete and the residual generator designed would not have been of lowest possible order in the case of systems with non-controllable stable modes.

\section{References}

[1] The Polynomial Toolbox 2.5. Polyx, Czech Republic. URL: http://www.polyx.com, 2001.

[2] J. Chen and R. J Patton. Robust Model-Based Fault Diagnosis for Dynamic Systems. Kluwer Academic Publishers, 1999.

[3] E.Y. Chow and A.S. Willsky. Analytical redundancy and the design of robust failure detection systems. IEEE Trans. on Automatic Control, 29(7):603-614, 1984.

[4] X. Ding and P.M. Frank. Frequency domain approach and threshold selector for robust model-based fault detection and isolation. IFAC Fault Detection, Supervision and Safety for Technical Processes, pages 271-276, Baden-Baden, Germany, 1991.

[5] E. Frisk and M. Nyberg. A minimal polynomial basis solution to residual generation for fault diagnosis in linear systems. Automatica, 37:1417-1424, 2001.

[6] J. Gertler. Fault Detection and Diagnosis in Engineering Systems. Marcel Dekker, 1998. 
[7] D. Henrion and M. Sebek. An algorithm for polynomial matrix factor extraction. International Journal of Control, 73(8):686-695, 2000.

[8] M. Hou. Fault detection and isolation for descriptor systems. In R.J. Patton, P.M. Frank, and R.N. Clark, editors, Issues of Fault Diagnosis for Dynamic Systems, chapter 5. Springer, 2000.

[9] M. Hou and P.C. Müller. Tracking control for a class of descriptor systems. In Proc. 13th IFAC World Congress, pages 109-114, San Fransisco, USA, 1996.

[10] Thomas Kailath. Linear Systems. Prentice-Hall, 1980.

[11] F. Kratz, S. Bousghiri, and W. Nuninger. A finite memory observer structure of continuous descriptor systems. In Proc. American Control Conference, pages 3900-3904, Seattle, USA, 1995.

[12] D. Maquin, B. Gaddouna, and J. Ragot. Generation of parity equations for singular systems. application to diagnosis. In Proceedings. International Conference on Systems, Man and Cybernetics., volume 3, pages 400-405, 1993.

[13] B. Marx, D. Koenig, and D. Georges. Robust fault diagnosis for linear descritor systems using proportional integral observers. In Proceedings. IEEE Conference on Decision and Control, volume 3, pages 457-462, Hawaii, USA, 2003.

[14] S.E. Mattson, H. Elmqvist, and M. Otter. Physical system modeling with modelica. Control Engineering Practice, 6(4):501-510, 1998.

[15] R. Nikoukhah. Innovations generation in the presence of unknown inputs: Application to robust failure detection. Automatica, 30(12):1851-1867, 1994.

[16] M. Nyberg. Criterions for detectability and strong detectability of faults in linear systems. International Journal of Control, 75(7):490-501, 2002.

[17] R.J. Patton, P.M. Frank, and R.N. Clark, editors. Issues of Fault Diagnosis for Dynamic Systems. Springer, 2000.

[18] J.W. Polderman and J.C. Willems. Introduction to Mathematical Systems Theory: A Behavioral Approach. New York: Springer Verlag, 1997.

[19] D. Sauter, H. Noura, F. Hamelin, and D. Theilliol. Parity space approach for fault diagnosis in descriptor systems. In In Proceedings CESA '06 IMACS Multiconference. Computational Engineering in Systems Applications, volume 1, pages 380-383, 1996.

[20] D.N. Shields. Robust fault detection for generalized state space systems. In International Conference on Control, pages 1335-1339, 1994.

[21] A. Varga. On computing least order fault detectors using rational nullspace bases. Safeprocess'2003, pages 229-234, Washington, USA, 2003. 
[22] N. Viswanadham, J.H. Taylor, and E.C. Luce. A frequency-domain approach to failure detection and isolation with application to GE-21 turbine engine control systems. Control - Theory and advanced technology, 3(1):4572, March 1987.

\section{A Proofs and Lemmas}

This section includes technical lemmas used in the proofs of the main theorems of the report.

In the proofs to follow, the set of all $z \in \mathcal{M}$ will, instead of (5), be characterized by using a differential equation, sometimes referred to as a kernel representation of the behavior. This is summarized in the following lemma, adapted from [18].

\section{Lemma 1.}

$$
\mathcal{M}=\left\{z \mid N_{H}(p) L(p) z=0\right\}
$$

where $N_{H}(s)$ is a minimal polynomial basis for the left null-space of $H(s)$.

The following lemma proves that, when $z \in \mathcal{M}$, a residual will be smooth.

Lemma 2. If $R(s)$ is a residual generator, $z \in \mathcal{M}$, and $r$ fulfills $r=R(p) z$, then $r$ can be written as

$$
r(t)=\sum_{i} q_{i}(t) e^{c_{i} t}
$$

where $q_{i}$ are polynomials in $t$.

Proof. The fact that $z \in \mathcal{M}$ is according to Lemma 1 equivalent to that $z$ fulfills

$$
Q(p) z=0
$$

where $Q(s)=N_{H}(s) L(s)$. Since $Q(s)$ has full rank, we can partition $Q(s)$ into $\left[Q_{1}(s) Q_{2}(s)\right]$ such that $Q_{1}(s)$ is square and has full rank. Also let $K_{1}, K_{2}, z_{1}$, $z_{2}$ correspond to the partition of $Q(s)$. Since $Q_{1}(s)$ is square and full rank, for any choice of $z_{2}$, there will always be a $z_{1}$ such that (18) is fulfilled.

Now choose an arbitrary $z_{2}$. Then there exist a $z_{1}$ such that $z \in \mathcal{M}$ where $z=\left[\begin{array}{ll}z_{1}^{T} & z_{2}^{T}\end{array}\right]^{T}$. Let $r$ be a signal satisfying $r=R(p) z$. This means that

$$
\left[\begin{array}{c}
d(p) \\
0
\end{array}\right] r=\left[\begin{array}{l}
K_{1}(p) \\
Q_{1}(p)
\end{array}\right] z_{1}+\left[\begin{array}{l}
K_{2}(p) \\
Q_{2}(p)
\end{array}\right] z_{2}
$$

By multiplying (19) with the row-vector $[n(s) M(s)]$ from the left, where

$$
[n(s) M(s)]\left[\begin{array}{l}
K_{1}(s) \\
Q_{1}(s)
\end{array}\right]=0
$$

we obtain

$$
n(p) d(p) r=\left(n(p) K_{2}(p)+M(p) Q_{2}(p)\right) z_{2}
$$

Using the definition of residual generator together with the facts that $z \in \mathcal{M}$ and $r=R(p) z$ implies that $r(t) \rightarrow 0$. In summary, we have shown that for all $z_{2}$, there exists an $r$ fulfilling (21) where $r(t) \rightarrow 0$. It can then be realized that it must hold that $n(s) K_{2}(s)+M(s) Q_{2}(s)=0$. 
For the $z$ that is given in the condition of the lemma, take out the $z_{2}$-part. The $z_{1}$-part will, together with $z_{2}$, then fulfill (18). Further, the given $r$ will fulfill (21), and therefore also $n(p) d(p) r=0$. Thus, $r$ has been proven to be a solution to the differential equation $n(p) d(p) r=0$. From this, together with the fact that the functional class considered for solutions is piece-wise continuous functions with finitely many impulses, it follows that $r$ can be written as

$$
r(t)=\sum_{i} q_{i}(t) e^{c_{i} t}
$$

where $q_{i}$ are polynomials in $t$ which ends the proof.

Lemma 3. A proper filter $R(p)$ with transfer operator $R(p)=d^{-1}(p) K(p)$ is a residual generator if and only if for all $z \in \mathcal{M}$ it holds that $\lim _{t \rightarrow \infty} K(p) z(t)=$ 0 .

Proof. For the only-if part, we assume that $z \in \mathcal{M}$, and according to Lemma 2, all solutions $r$ to $d(p) r=K(p) z$ can then be written as (17). Further, since $R(s)=K(s) / d(s)$ is a residual generator, it holds that $\lim _{t \rightarrow \infty} r(t)=0$. Therefore all nonzero parts (i.e. $q_{i} \neq 0$ ) of the sum (17) are stable. All orders of derivatives of $r$ are trivially also stable. Thus $d(p) r(t) \rightarrow 0$, and also $K(p) z(t) \rightarrow 0$, when $t \rightarrow \infty$.

For the if-part, we know that $K(p) z(t) \rightarrow 0$ and that the signal $K(p) z(t)$ is filtered through a stable filter (i.e. $d(s)$ is stable). Therefore, it must hold that also $r(t) \rightarrow 0$.

Lemma 4. Let the row-vector $K(p)$ satisfy $z \in \mathcal{M} \Rightarrow \lim _{t \rightarrow \infty} K(p) z(t)=0$. Then there exists a polynomial row-vector $\gamma(s)$ such that $K(s)=\gamma(s) Q_{r}(s)$.

Proof. First we prove that there exists a rational $\phi(s)=b^{-1}(s) n(s)$ such that $K(s)=\phi(s) Q_{r}(s)$. For this, let $U(s)$ be a matrix whose columns form a polynomial basis for the right null-space of $Q_{r}(s)$. Then, for any vector and also $K(s)$, it holds that there exists a scalar polynomial $b(s)$ and polynomial row-vectors $n(s)$ and $m(s)$ such that $b(s) K(s)=n(s) Q_{r}(s)+m(s) U^{T}(s)$. This implies that $b(s) K(s) U(s)=m(s) U^{T}(s) U(s)$ and from the definition of $U(s)$ it follows that $Q(p) U(p) \nu=0$ for all $\nu(t)$. Equation (16) and the assumption in the lemma formulation gives that $\forall \nu: K(p) U(p) \nu \rightarrow 0$ when $t \rightarrow \infty$. It then follows that $K(p) U(p)=0$ which implies that $m(s) U^{T}(s) U(s)=0$. Since $U(s)$ has full column rank this implies that $m(s)=0$ which ends the proof of the existence of a rational $\phi(s)$.

Now, given such a rational $\phi(s)=\frac{n(s)}{b(s)}$ such that $K(s)=\phi(s) Q_{r}(s)$ where the polynomial $b(s)$ and the polynomial row-vector $n(s)$ are co-prime. Assume that $\phi(s)$ is not polynomial, i.e. $b(s)$ has a zero at $s=s_{0}$. Then

$$
n(s) Q_{r}(s)=b(s) K(s)
$$

Since $n(s)$ and $b(s)$ are co-prime, it holds that $n\left(s_{0}\right) \neq 0$. Therefore, $n\left(s_{0}\right) \neq 0$ must reside in the left null-space of $Q_{r}\left(s_{0}\right)$. By definition it follows that $Q_{r}(s)$ has no stable zeros and from the assumption that $[H(s) L(s)]$ has full row rank it follows that $Q_{r}(s)$ has full row rank. This means that $Q_{r}(s)$ can only lose rank if $s=s_{0}$ is an unstable zero, i.e. $\operatorname{Re}\left\{s_{0}\right\} \geq 0$. Thus, if $b(s)$ has a zero $s=s_{0}$, it must be an unstable zero. 
Therefore assume that $s_{0}$ is an arbitrary unstable zero of $b(s)$. By using the Smith form, $Q_{r}(s)$ can be written

$$
Q_{r}(s)=U(s)\left[\operatorname{diag}\left(\epsilon_{1}(s), \ldots, \epsilon_{n-1}(s), \epsilon_{n}(s)\right) \quad 0\right] V(s)
$$

where $U(s)$ and $V(s)$ are unimodular. Then (22) can be written as $n(s) U(s) S(s)=$ $b(s) K(s) V^{-1}(s)$. This means that $n\left(s_{0}\right) U\left(s_{0}\right) S\left(s_{0}\right)=0$. Because of the coprimeness, $n\left(s_{0}\right)$ must be nonzero. Further since $U\left(s_{0}\right)$ has full rank, also the vector $n\left(s_{0}\right) U\left(s_{0}\right)$ must be nonzero. Let $i$ be the position of one nonzero element $\alpha_{i}$ in $n\left(s_{0}\right) U\left(s_{0}\right)$. Then

$$
0=n\left(s_{0}\right) U\left(s_{0}\right) S\left(s_{0}\right)=\left[\ldots \alpha_{i} \ldots\right] S\left(s_{0}\right)=\left[\ldots \alpha_{i} \epsilon_{i}\left(s_{0}\right) \ldots\right]
$$

Thus $\alpha_{i} \epsilon_{i}\left(s_{0}\right)=0$ which means that $\epsilon_{i}(s)$ must have a zero in $s=s_{0}$, i.e. $\epsilon_{i}(s)=\epsilon_{i}^{\prime}(s)\left(s-s_{0}\right)$. The polynomial $\epsilon_{i}^{\prime}(s)$ can also contain the factor $\left(s-s_{0}\right)$. Let the multiplicity of this factor be $\mu$, i.e. $\epsilon_{i}^{\prime}(s)=\epsilon_{i}^{\prime \prime}(s)\left(s-s_{0}\right)^{\mu}$.

Next choose a solution $\bar{z}_{i}$ in accordance with Lemma 5 . This means that $\left(s-s_{0}\right)^{\mu+1} \bar{z}_{i}=0$. Now define $\bar{z}=\left[0 \ldots 0, \bar{z}_{i}, 0, \ldots, 0\right]^{T}$, and choose $z$ as $z=$ $V^{-1}(p) \bar{z}$. Next note that $Q_{r}(p) z=U(p) S(p) V(p) V^{-1}(p) \bar{z}=U(p) S(p) \bar{z}$. Since $\epsilon_{i}(s)=\epsilon_{i}^{\prime \prime}(s)\left(s-s_{0}\right)^{\mu+1}$ and $\bar{z}_{i}$ is a solution to $\left(s-s_{0}\right)^{\mu+1} \bar{z}_{i}=0$, it holds that $S(p) \bar{z}=0$ and thus, $Q_{r}(p) z=0$ and also $Q(p) z=0$.

By factorizing $b(s)$ as $b(s)=b^{\prime}(s)\left(s-s_{0}\right)$, the $i$ :th element of the vector equation $n(s) U(s) S(s)=b(s) K(s) V^{-1}(s)$ can be written

$$
n(s) U_{i}(s) \epsilon_{i}^{\prime}(s)\left(s-s_{0}\right)=b^{\prime}(s)\left(s-s_{0}\right)\left[K(s) V^{-1}(s)\right]_{i}
$$

where $U_{i}(s)$ is the $i$ :th column of $U(s)$. Cancelling $\left(s-s_{0}\right)$ on both sides results in

$$
n(s) U_{i}(s) \epsilon_{i}^{\prime \prime}(s)\left(s-s_{0}\right)^{\mu}=b^{\prime}(s)\left[K(s) V^{-1}(s)\right]_{i}
$$

Since $\alpha_{i} \neq 0, n(s) U_{i}(s)$ has no zero in $s=s_{0}$. Therefore it follows from Lemma 5 that $n(p) U_{i}(p) \epsilon_{i}^{\prime \prime}(p)\left(p-s_{0}\right)^{\mu} \bar{z}_{i} \nrightarrow 0$. Using (26) gives that $b^{\prime}(p) K(p) V^{-1}(p) \bar{z} \not \rightarrow$ 0 and further that $b^{\prime}(p) K(p) z \nrightarrow 0$. This means that $K(p) z \nrightarrow 0$. Thus we have proven that there exists a $z \in \mathcal{M}$ such that $K(p) z(t) \nrightarrow 0$. This is a contradiction and thus $b(s)$ can not have an unstable zero.

Lemma 5. If $s=s_{0}$ is an unstable zero, there exists a solution $\bar{z}_{i}$ to the differential equation $\left(p-s_{0}\right)^{\mu+1} \bar{z}_{i}=0$ such that for all polynomials $\beta(s)$ with no zero in $s=s_{0}$ it holds that $\beta(p)\left(p-s_{0}\right)^{\mu} \bar{z}_{i} \nrightarrow 0$.

Proof. Let $\bar{z}_{i}=t^{\mu} e^{s_{0} t}$. Its Laplace transform is $\frac{\mu !}{\left(s-s_{0}\right)^{(\mu+1)}}$. Then the Laplace transform of the signal $v=\left(p-s_{0}\right)^{\mu} \bar{z}_{i}$ is

$$
V(s)=\frac{\mu !\left(s-s_{0}\right)^{\mu}}{\left(s-s_{0}\right)^{(\mu+1)}}=\frac{\mu !}{\left(s-s_{0}\right)}
$$

Inverse Laplace transform gives that $v=\mu ! e^{s_{0} t}$. Now take an arbitrary polynomial $\beta(s)$ with no zero in $s=s_{0}$. Then $\beta(p) v=\beta\left(s_{0}\right) \mu ! e^{s_{0} t}$. Since $s=s_{0}$ is an unstable zero, and $\beta\left(s_{0}\right) \neq 0$, it holds that $\beta(p) v \nrightarrow 0$. 